\title{
Power/Ground Network Aware and Row- Based Solutions to the Crosstalk Driven Routing Problem *
}

\author{
Jinghong Liang, Tong Jing, Xianlong Hong \\ Computer Science and Technology Department \\ Tsinghua University \\ Beijing 100084, P. R. China \\ Phone: +86-10-62785564 \\ Fax: +86-10-62781489 \\ E-mail: liangjh03@mails.tsinghua.edu.cn \\ \{jingtong,hxl-dcs\}@tsinghua.edu.cn
}

\author{
Jinjun Xiong, Lei He \\ Electrical Engineering Department \\ UCLA \\ Los Angeles, California 90095-1594, USA \\ Phone: (310) 206-2037 \\ Fax: (310) 206-4685 \\ Email: jinjun@ucla.edu \\ lhe@ee.ucla.edu
}

\begin{abstract}
This paper gives power/ground network aware and row-based solutions to the crosstalk driven routing problem. Routability and timing issues are also considered. The algorithm has been implemented and tested on MCNC benchmarks. Compared with the recent work introduced in [11], the proposed algorithm can achieve more than $72 \%$ on average improvements for the adjacent edges while considering power/ground network, which greatly reduces potential vias.
\end{abstract}

\section{INTRODUCTION}

Chip design is with increasingly shrinking geometries yet increasingly higher frequencies. Coupling effects and crosstalk have gained more concerns for chip performance optimization [1]. Crosstalk elimination can be performed in two different ways. One is with the circuit design method, such as adding suitable capacitances or inserting buffers. The other is with the help of CAD tools, such as routing tool in the physical design phase. So, crosstalk elimination has become a challenge to chip routing.

Some existing works focus on coupling noise and crosstalk elimination in the routing phase, which mainly fall into three categories, noise modeling [2], [3], noise minimization [4]-[7], and simultaneous noise minimization and performance optimization [8]-[11]. [2] derived expressions for a coupling capacitance and a crosstalk voltage height. [3] declared that on-chip inductance, especially mutual inductance, should be considered for high-performance interconnect design and presented the effective coupling model, called the $K_{\text {eff }}$ model. [4] described a two-pass algorithm including region-based crosstalk risk estimation and crosstalk reduction. [5] presented a cost function taking crosstalk into consideration, and is used during the phase of constructing the routing tree. [6] proposed

* This work was supported in part by the NSFC under Grant (China) No.60373012, the NSF CAREER Award (USA) CCR-0401682, the SRFDP of China under Grant No.20020003008, and the Hi-Tech Research and Development (863) Program of China under Grant No.2004AA1Z1050. a three-phase algorithm based on crosstalk budgeting, simultaneous shield insertion and net ordering (SINO), and local refinement. Both [8] and [9] proposed performance optimization global routing algorithms considering crosstalk reduction. The former mainly focuses on coupling capacitance and uses spacing method. The latter considers coupling inductance and is based on shield insertion. [10] presented an efficient RLC crosstalk reduction algorithm by using Tabu search method and achieved about 20x speedup compared with [9]. The latest progress is the global routing algorithm, AT-PO-GR, to minimize the routing area under congestion, timing, and RLC crosstalk constraints [11].

However, [9]-[11] did not take actual power/ground (P/G) network and potential via minimization into consideration. The main contribution of this paper is the $\mathrm{P} / \mathrm{G}$ network aware and row-based solutions to the crosstalk driven routing problem. This algorithm (1) takes $\mathrm{P} / \mathrm{G}$ network into consideration to meet the practical applications, (2) and gives a row-based routing solution to minimize potential vias, which is useful for good manufacturability.

The remainder of this paper is organized as follows. Section II formulates the routing problem considering $\mathrm{P} / \mathrm{G}$ network. In Section III, we discuss the routing algorithm in detail. Section IV shows experimental results and discussions. Section V concludes the whole work and gives some possibilities for future work.

\section{PROBLEM FORMULATION}

\section{A. GRG with $P / G$ network}

With the progress in multi-layer VLSI routing technology, routing area is a whole chip plane. Thus, a net can be specified as a set of nodes in global routing graph (GRG). Then, the problem of routing a net can be described as a rectilinear Steiner tree (RST) problem of specified nodes in GRG [12].

But generally, while we mapping the $\mathrm{P} / \mathrm{G}$ network on the 
GRG, we'll not get the same/similar grid in the graph. Fig.1 shows the general relationship between the mapping of $\mathrm{P} / \mathrm{G}$ network and the grid in GRG. We can see that $\mathrm{P} / \mathrm{G}$ mapping is sparse than GRG grid. Then, while performing crosstalk elimination with shielding, we should consider this problem, here we call it the incongruous grid problem, which limits the shielding style and shielding number. We can only get fewer horizontal shields and more vertical ones. And the horizontal shields are longer ones relative to one GRG grid.

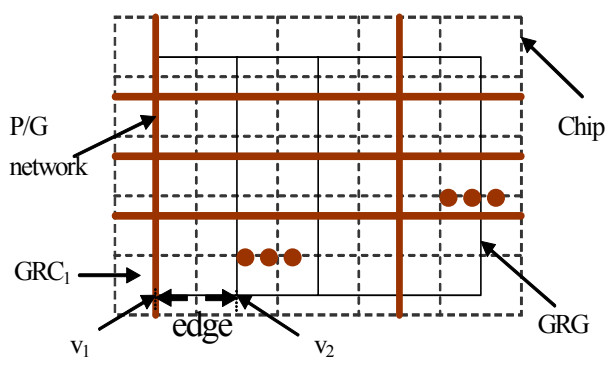

Fig.1. GRG with $\mathrm{P} / \mathrm{G}$ network.

\section{B. RLC Noise Model}

The LSK model for RLC crosstalk [3], [7] is used in this paper. Different from earlier noise model [2], the LSK model considers coupling inductance between adjacent and non-adjacent sensitive nets. A formula-based $K_{\text {eff }}$ model has been developed in [3] to calculate the inductive coupling coefficient $k_{i t, j t}$. Furthermore, the total amount of inductive coupling induced on segment $N_{i t}$ can be represented by the sum of the inductive coupling coefficient $K_{i t}=\sum_{j \neq i} k_{i t, j t}$ for all net segments $N_{j t}$ that are sensitive to $N_{i t}$. To consider the effect of interconnect length and the general case where the total coupling is not uniform in all routing regions, a length-scaled $K_{\text {eff }}(L S K)$ model was proposed in [7], where the $L S K$ value is defined as $L S K=\sum_{t} l_{t} \cdot K_{i t}$, where $l_{t}$ is length of $R_{t}$ and $K_{i t}$ is total coupling for $N_{i t}$ in region $t$.

\section{Problem Formulation}

Let

$$
S_{i t}= \begin{cases}1, & \text { edge } t \text { be used by net } i, \\ 0, & \text { otherwise. }\end{cases}
$$

where $S_{i t}$ is a kind of stamps indicating whether edge $t$ contains net $i$.

$$
\begin{aligned}
& \text { Then we have } \\
& \text { Minimize } \\
& \text { Subject to } \\
& L=\sum_{i=1}^{N_{n}} \sum_{t=1}^{N_{e}} S_{i t} l_{t} \\
& f_{t}=\sum_{i=1}^{N_{n}} S_{i t}+s n_{t}+o_{t} \leq c_{t}, \forall t \in E ; \\
& T(i, j) \leq T_{D}(i, j), \forall i \in N, \forall j \in s(i) \text {; }
\end{aligned}
$$

$$
L S K_{i j} \leq \overline{L S K_{i j}}, \forall i \in N, \forall j \in s(i)
$$

Formula (2) is the congestion constraint, which forbids the overflow on each GRG edge. Formula (3) guarantees the actual delay value from source $i$ to $\operatorname{sink} j, T(i, j)$, is no more than the given timing constraint $T_{D}(i, j)$. Formula (4) sets the upper bound of $L S K, \overline{L S K_{i j}}$, for each source sink pair $i j$. The actual $L S K$ value of this pair, $L S K_{i j}$, could not exceed the bound.

\section{OUR Routing Algorithm}

\section{A. Incongruous Grid Problem}

Fig. 2 shows a local part of GRG. We can see 3 GRG edges, edge60, edge61, and edge62. Fig.3 shows an example of previous routing result. Brown lines denote the shields, black lines denote the net segments, and dashed lines are unused tracks. Since shields provided by $\mathrm{P} / \mathrm{G}$ network are as long as three GRG edges, the previous routing results should be improved to meet the practical applications. W can adjust orders of the horizontal net segments shown in Fig. 4.

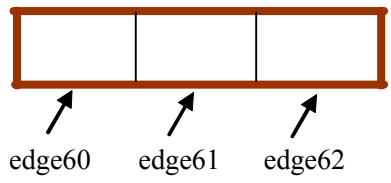

Fig. 2. A local $\mathrm{P} / \mathrm{G}$ network and GRG edges.

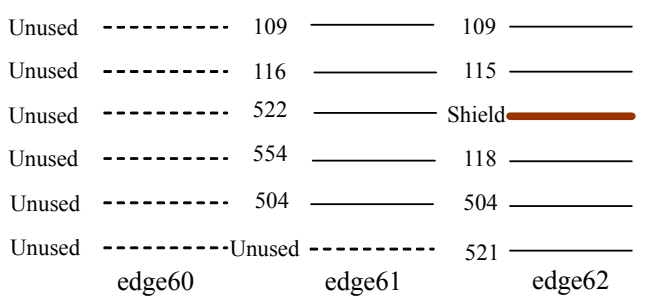

Fig. 3. An example of previous routing result.

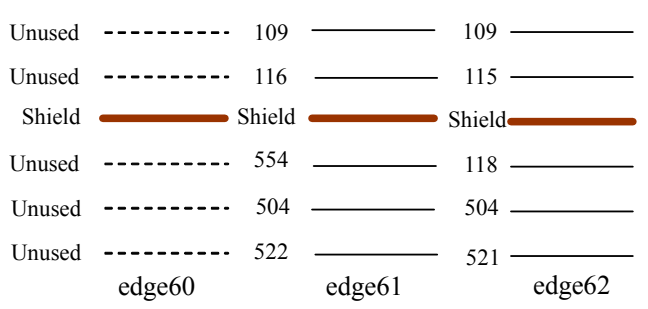

Fig. 4. The result after adjusting.

\section{B. Row-Based Solution}

Previous routing algorithms [9]-[11] performed crosstalk elimination only in single GRG edge. So, the routing results cause "dog-leg", i.e., segments of the same net in adjacent 
GRG edges but are in different tracks (denoted by the order in the global routing phase), which makes more potential vias in detailed routing. Fig.5 shows an example. Two segments of Net1 are not in the same track. We try to find a row-based solution to tackle this "dog-leg" problem in this Section.

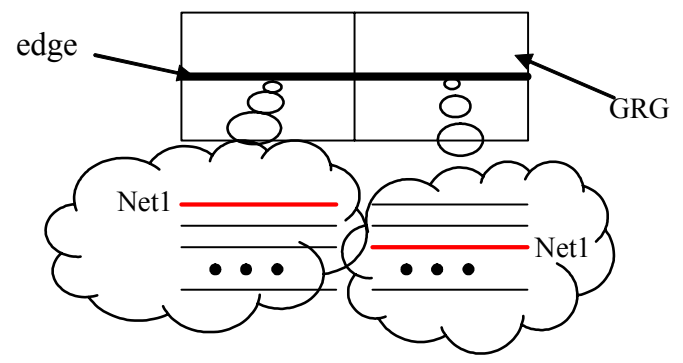

Fig. 5. The "dog-leg" problem.

\section{Our Methods}

1. Obtain Row-Based Solution

We still use Tabu search method [9] to eliminate crosstalk noise. But we use a new cost formula of a GRG edge as follows to take the "dog-leg" problem into consideration.

$$
\operatorname{cost}(x)=w_{1} c_{1}+w_{2} c_{2}+w_{3} c_{3}+w_{4} c_{4}+w_{5} c_{5}
$$

where $w_{1}, w_{2}, w_{3}$, and $w_{4}$ are the weights that equal to 13,2 , 13 , and 10 , respectively. $c_{1}$ is the number of adjacency of sensitivity rate, $c_{2}$ is the sum of violation $K_{\text {eff }}$ value, $c_{3}$ is the number of $K_{\text {eff }}$ violation, and $c_{4}$ is the number of shield inserted. $w_{5}$ equals $15, c_{5}=(1-a / b), a$ is the number of the adjacent segments of a net in the same tracks, $b$ is the capacity of the GRG edge.

2. Tackle Incongruous Grid Problem

Aware of $\mathrm{P} / \mathrm{G}$ network, we assume that the horizontal shields are as long as 3 GRG edges. We try to make the 3 adjacent horizontal GRG edges have the same shield order by using the following method.

Firstly, we partition all the horizontal GRG edges into edge groups, each of which includes 3 adjacent edges. Then, in the same edge group, we can get the critical edge, which has the largest shield number. After that, we let the same shields track (i.e., the same shield order) in the other two edges (non-critical edges). At last, we can adjust the segments in the non-critical edges to make use of the assigned shields. Formula (5) is also used here to minimize the cost. Fig.4 shows an example. Edge62 in Fig. 4 is the critical edge. Then, we add one shield as the same order in edge60 and edge61, respectively. After that, we adjust the segments in these two edges. At last, we got the minimal cost routing solution shown in Fig.4.

\section{EXPERIMENTAL RESULTS AND DisCUSSIONS}

Our algorithm has been implemented in $\mathrm{C}$ language. It performs on a SUN V880 workstation with Unix OS. We compared our results with that of AT-PO-GR [11].

\section{A. Benchmark Data}

We tested 5 MCNC benchmarks given in TABLE I.

TABLE 1

BENCHMARK DATA

\begin{tabular}{|l|l|l|}
\hline Circuits & Number of net & Grids \\
\hline C2 & 745 & $9 * 11$ \\
\hline C5 & 1764 & $16 * 18$ \\
\hline C7 & 2536 & $16 * 18$ \\
\hline S13207 & 4953 & $24 * 26$ \\
\hline Avq & 21581 & $65 * 67$ \\
\hline
\end{tabular}

\section{B. Results and Discussions}

$\mathrm{RB}$ denotes the algorithm to get a row-based solution and IG denoted the algorithm considering incongruous grid problem.

TABLE 2

Comparsion of Number of Adjacent Edges Have Same Track Position

\begin{tabular}{|l|l|l|l|}
\hline Circuits & AT-PO-GR & \multicolumn{1}{|c|}{ RB } & \multicolumn{1}{c|}{ IG } \\
\hline C2 & 116 & 339 & 202 \\
\hline C5 & 361 & 413 & 654 \\
\hline C7 & 382 & 416 & 797 \\
\hline S13207 & 1382 & 2322 & 4770 \\
\hline Avq & 3207 & 5793 & 12513 \\
\hline Aver imp & ------ & $72.84 \%$ & $159.85 \%$ \\
\hline
\end{tabular}

TABLE 3

COMPARSION OF TOTAL WiRE LENGTH

\begin{tabular}{|l|l|l|l|}
\hline Circuits & AT-PO-GR & RB & IG \\
\hline C2 & 462204 & 465886 & 465886 \\
\hline C5 & 1320742 & 1327700 & 1327700 \\
\hline C7 & 1516366 & 1520446 & 1520446 \\
\hline S13207 & 9881044 & 9894684 & 9894684 \\
\hline Avq & 9899034 & 9873887 & 9873887 \\
\hline Aver imp & ----- & $0.29 \%$ & $0.29 \%$ \\
\hline
\end{tabular}

TABLE 4

Comparsion of TOtal RunNing Time

\begin{tabular}{|l|l|l|l|}
\hline Circuits & AT-PO-GR & \multicolumn{1}{c|}{ RB } & IG \\
\hline C2 & 87.21 & 70.44 & 98.08 \\
\hline C5 & 257.93 & 212.20 & 275.13 \\
\hline C7 & 331.25 & 279.21 & 345.41 \\
\hline S13207 & 2049.16 & 1436.15 & 1822.97 \\
\hline Avq & 6171.94 & 5612.33 & 6479.56 \\
\hline Aver imp & ------ & $18.33 \%$ & $-3.47 \%$ \\
\hline
\end{tabular}

TABLE 5

COMPARSION OF TOTAL AREA

\begin{tabular}{|l|l|l|l|}
\multicolumn{5}{c}{ COMPARSION of TOTAL AREA } \\
\hline Circuits & AT-PO-GR & \multicolumn{1}{c|}{ RB } & IG \\
\hline C2 & $168 * 204$ & $174 * 203$ & $175 * 203$ \\
\hline C5 & $304 * 332$ & $301 * 333$ & $302 * 333$ \\
\hline C7 & $342 * 365$ & $360 * 377$ & $364 * 377$ \\
\hline S13207 & $1208 * 1410$ & $1203 * 1420$ & $1216 * 1420$ \\
\hline Avq & $1216 * 1001$ & $1215 * 1003$ & $1223 * 1003$ \\
\hline Aver imp & ------ & $-2.30 \%$ & $-3.08 \%$ \\
\hline
\end{tabular}

1. We compare the number of adjacent net segments (in the same track order) in TABLE 2. It clearly shows that RB improves the adjacent edges by $72.84 \%$ on average 
compared to that of AT-PO-GR, which indicates the effectiveness of our methods in aligning routing solutions, thus reducing potential doglegs and vias for detailed routing. We also note that $\mathrm{IG}$ is on average $159.85 \%$ more than that of AT-PO-GR. This is because we add empty tracks and have more possibility to adjust the net segments.

2. As shown in TABLE 3, the total wire length of RB and IG are almost the same as that of AT-PO-GR because our goal is to minimize the wire length.

3. According to TABLE 4, the running time of $\mathrm{RB}$ is slightly shorter than that of AT-PO-GR because the area is larger and we are more flexible in routing. The running time of IG is about $20 \%$ longer than that of RB because we add a long shield adjusting process.

4. From TABLE 5, we can see that the area of RB is about $3 \%-8 \%$ larger than that of AT-PO-GR. That is because we use new cost computation formula, the area owns a smaller proportion than previous. The area of IG is slightly larger than that of BR because in some cases, the maximal track number of a GRG edge may increase after long shield adjusting (see Section C).

\section{An Example}

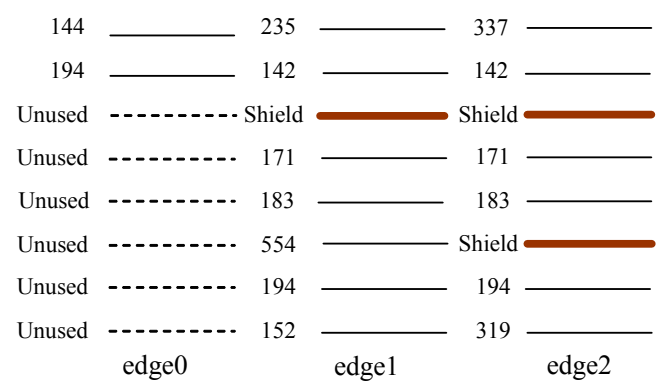

Fig.6. GRG edges before long shield adjusting.

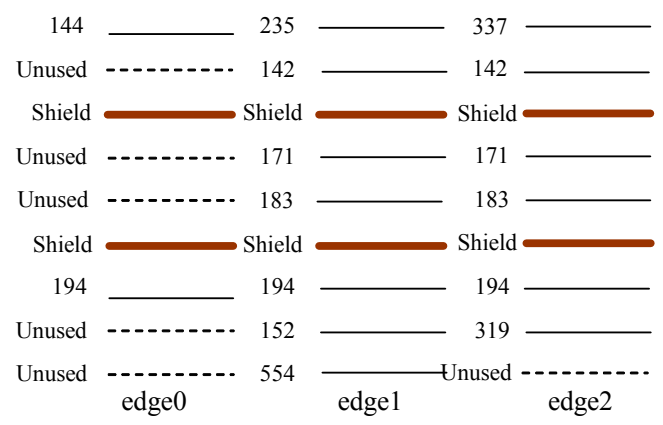

Fig.7. The long shield adjusting result.

We can see that the critical edge in Fig. 6 is edge2, and edge 1 has the maximal height of 8 . But when we add a shield into edge1, the height of it must increase 1. From Fig.7, we can see that the new maximal height is 9. If the maximal height of a row increases, the total area will increase.

In Fig.6, we can see that only the segments of edge1 have the possibility to be move to the same position as the edge 0 . But in Fig.7, in long shield adjusting process, the empty tracks of the left could be used. So we can move the segment 194 to the same position as the edge1. So the number of net segments in adjacent edges have the same track position increases.

\section{CONCLUSIONS AND FUTURE WORK}

A performance and RLC crosstalk driven routing algorithm considering $\mathrm{P} / \mathrm{G}$ network and row-based solution is presented in this paper. The experimental results show this algorithm can (1) take $P / G$ network into consideration to meet the practical applications, (2) and can give a row-based routing solution to minimize potential vias, which is useful for good manufacturability.

As our future work, we plan to make our algorithm more practical to real chip routing, and design better strategies for crosstalk elimination.

\section{REFERENCES}

[1] T. Jing, X. L. Hong. "The Key Technologies of Performance Optimization for Nanometer Routing”. In: Proc. IEEE ASICON, Beijing, China, 2003, pp.118-123.

[2] T. Sakurai, S. Kobayashi, and M. Node. "Simple expressions for interconnecting delay, coupling and crosstalk in VLSI's", In: Proc. IEEE ISCAS, Singapore, 1991, pp.2375-2378.

[3] L. He and K. M. Lepak. "Simultaneous shield insertion and net ordering for capacitive and inductive coupling minimization", In: Proc. ACM ISPD, San Diego, CA, USA, 2000, pp.56-61.

[4] T. X. Xue, E. S. Kuh, and D. S. Wang. "Post global routing crosstalk synthesis", IEEE Trans on CAD, 1997, 16(12): pp.1418-1430.

[5] J. J. Xiong and L. He. "Full-Chip Routing Optimization With RLC Crosstalk Budgeting", IEEE Trans on CAD, 2004, 23(3) pp. 366-377.

[6] H. Zhou and D. F. Wong. "Global routing with crosstalk constraints", IEEE Trans on CAD, 1999, 18(11): pp.1683-1688.

[7] J. J. Xiong and L. He, "Extended Global Routing with RLC Crosstalk Constraints", IEEE Trans on Very Large Scale Integration Systems. 2005, 13(3): pp.319-329.

[8] J. Y. Xu, X. L. Hong, T. Jing, L. Zhang, J. Gu. "A Coupling and Crosstalk Considered Timing-Driven Global Routing Algorithm for High Performance Circuit Design", In: Proc. IEEE/ACM ASP-DAC, 2004, Yokohama, Japan, pp.677-682.

[9] L. Zhang, T. Jing, X. L. Hong, J. Y. Xu, J. J. Xiong, L. He. "Performance Optimization Global Routing with RLC Crosstalk Constraints", In: Proc. IEEE ASICON, Beijing, China, 2003, pp.191-194.

[10] L. Zhang, T. Jing, X. L. Hong, J. Y. Xu, J. J. Xiong, L. He. "Performance and RLC Crosstalk Driven Global Routing", In: Proc. IEEE ISCAS, 2004, Vancouver, Canada, pp.V65-68.

[11] T. Jing, L. Zhang, J. H. Liang, J. Y. Xu, X. L. Hong, J. J. Xiong, L. He. "A Min-Area Solution to Performance and RLC Crosstalk Driven Global Routing Problem", In: Proc. IEEE/ACM ASP-DAC, 2005, Shanghai, China, pp.115-120.

[12] T. Jing, X. L. Hong, J. Y. Xu, H. Y. Bao, C. K. Cheng, and J. Gu. "UTACO: A Unified Timing and Congestion Optimization Algorithm for Standard Cell Global Routing." IEEE Trans on CAD. 2004, 23(3): pp.358-365. 